\title{
Sinopsis de la Situación de Salud Oral en Chile - Parte III: Encuestas Nacionales de Salud.
}

\section{Synopsis or the Oral Health Situation in Chile - Part III: National Health Surveys.}

\author{
Alicia Morales ${ }^{1,2^{*}}$, Nayib Hussein ${ }^{3}$, Jose Polanco ${ }^{1}$, Gisela Jara ${ }^{1,2}$, Fabiola Werlinger ${ }^{1,2}$, \\ Rodrigo Cabello ${ }^{1,2}$, Iris Espinoza ${ }^{1,2}$, Rodrigo Giacaman ${ }^{2,4}$, Ximena Lee ${ }^{1,2}$, Paola Carvajal',2, \\ Oscar Arteaga ${ }^{2,5}$, Franco Cavalla ${ }^{1,2}$, Camila Corral' ${ }^{1,2}$, Mauricio Baeza ${ }^{1,2}$, Gonzalo Rodríguez ${ }^{1,2}$, \\ Soraya León ${ }^{2,4}$, Karla Gambetta ${ }^{2,4}$, Jorge Gamonal ${ }^{1,2^{*}}$
}

\begin{abstract}
1. Facultad de Odontología, Universidad de Chile, Chile.

2. Centro de Vigilancia y Epidemiología de las

Enfermedades Orales - CEVEO, Chile.

3. CESFAM Francisco Boris Soler, Melipilla, Chile

4. Universidad de Talca, Chile

5. Escuela de Salud Pública, Universidad de Chile, Chile.
\end{abstract}

* Correspondencia Autor: Alicia Morales | Dirección: Sergio Livingstone 943, comuna de Independencia, Santiago, Chile. | Teléfono: (56) 2 29776772. | E-mail:amorales@odontologia.uchile.cl

Jorge Gamonal | Dirección: Sergio Livingstone 943, comuna de Independencia, Santiago, Chile. | Teléfono: (56) 2 29776772. | E-mail: jgamonal@ odontologia.uchile.cl

Trabajo recibido el 12/02/2020.

Aprobado para su publicación el 06/06/2020

\section{RESUMEN}

Este artículo realiza una revisión y síntesis de las principales encuestas poblacionales de salud en Chile. Se describen sus principales características y hallazgos con el objetivo de orientar a los profesionales odontólogos en el conocimiento de material existente para el diagnóstico odontológico objetivo, así como el impacto de la salud oral en la calidad de vida, a nivel poblacional.

\section{PALABRAS CLAVE:}

Salud bucal, encuestas poblacionales, prevalencia, epidemiología.

Int. J. Inter. Dent Vol. 13(3); 140-147, 2020.

\section{ABSTRACT}

This article makes a brief review and synthesis of national health surveys in Chile. The article describes the main characteristics and findings in order to guide dental professionals with the knowledge of the existing material for an objective population oral health diagnosis, as well as the impact of oral health on quality of life of the population. KEY WORDS:

Oral health; Health survey; Prevalence; Epidemiology.

Int. J. Inter. Dent Vol. 13(3); 140-147, 2020.

\section{INTRODUCCIÓN}

El proceso de reforma en salud que Chile inició el año 2000 ha requerido disponer de un diagnóstico actualizado para evaluar y reformular las políticas sectoriales, estimar la demanda que enfrentará el sistema de salud, y establecer la vigilancia epidemiológica correspondiente. El desarrollo de encuestas poblacionales ha permitido obtener información relevante para la planificación y programación sanitaria nacional(1). Dentro de este marco, se deben establecer diagnósticos poblacionales de las enfermedades sistémicas más prevalentes. Sin embargo, los signos clínicos de una enfermedad representan solo una parte del concepto de salud, integrándose actualmente la evaluación subjetiva del bienestar, permitiendo valorizar el impacto de la enfermedad y su tratamiento en la percepción del bienestar y la identificación de problemas de salud percibidos por la población ${ }^{(2)}$

El año 2000 el Ministerio de Salud (MINSAL) inició la Vigilancia de Enfermedades No Transmisibles y sus factores de riesgo (VENT), sumándose a las recomendaciones internacionales, desarrollando encuestas poblaciones, introduciendo primero la aplicación de cuestionarios, valorando aspectos subjetivos relacionados con la salud (Encuestas de Calidad de Vida y Salud 2000, 2006 y 2015-2016), para luego agregar la inclusión de mediciones poblacionales objetivas en las que se integra la utilización de cuestionarios y mediciones biofisiológicas y bioquímicas (Encuestas Nacionales de Salud 2003, 2009-2010, 20162017) $)^{(3)}$

El objetivo de este estudio fue realizar una revisión de la literatura con la finalidad de relevar la información sobre la salud oral de la población chilena contenida en las encuestas mencionadas anteriormente.

\section{MATERIALES Y MÉTODOS}

Se realizó una revisión narrativa para identificar encuestas de salud de base poblacional con representatividad nacional. Los criterios de inclusión consideraron estudios de base poblacional, con datos primarios, con el objetivo primario o secundario de establecer las condiciones de salud oral o el impacto de ésta en la calidad de vida relacionada con la salud oral. La búsqueda incluyó repositorios electrónicos científicos (Pubmed y Scielo) y fuentes de referencia nacional en salud (Ministerio de Salud) y universidades.

Los resultados obtenidos de la búsqueda se dividen en Caracterización de las Encuestas, Medición Objetiva de las Patologías Orales Más Prevalentes, Medición subjetiva del impacto de las patologías orales en la calidad de vida relacionada con la salud oral y Uso de Servicio Dental.

\section{Caracterización de las Encuestas:}

Se identificaron un total de seis estudios de representatividad nacional.

Se han realizado tres Encuestas Nacionales de Calidad de Vida y Salud (ENCAVI) (Tabla 1), y tres Encuestas Nacionales de Salud (ENS) (Tabla 2).

a. Primera Encuesta Nacional de Calidad de Vida y Salud 2000(4,5) (Tabla 1):

Teniendo como referencia la transición demográfica que apunta hacia el envejecimiento de la población, a fines del siglo XX, surge la necesidad de contar con estadísticas a nivel salud para generar respuestas apropiadas a través de políticas públicas. Bajo esta idea, surge en forma colaborativa entre el Instituto Nacional de Estadísticas (INE) y el MINSAL la ENCAVI 2000, cuyo objetivo principal fue establecer una línea base para la elaboración de planes y políticas de Promoción de Salud y 
Tabla 1. Encuesta Nacional de Calidad de Vida y Salud 2000, 2006, 2015 - 2016.

\begin{tabular}{|c|c|c|c|c|c|c|c|}
\hline Nombre & $\begin{array}{l}\text { Tipo de } \\
\text { estudio }\end{array}$ & $\begin{array}{c}\text { Población } \\
\text { objetivo }\end{array}$ & Autor & $\begin{array}{c}\text { Año de } \\
\text { desarrollo }\end{array}$ & $\begin{array}{c}\text { Tipo de } \\
\text { publicación }\end{array}$ & Objetivo & Evento de salud oral \\
\hline $\begin{array}{l}\text { Primera } \\
\text { Encuesta } \\
\text { Nacional de } \\
\text { Calidad de } \\
\text { Vida y Salud, } \\
2000\end{array}$ & $\begin{array}{l}\text { Encuesta } \\
\text { poblacional } \\
\text { de tipo trans- } \\
\text { versal. }\end{array}$ & $\begin{array}{l}\text { Personas } \geq 15 \\
\text { años ( } 6228 \\
\text { hogares) }\end{array}$ & MINSAL INE & 2000 & $\begin{array}{l}\text { Informe } \\
\text { técnico }\end{array}$ & $\begin{array}{l}\text { 1. Información para la elaboración de } \\
\text { planes y políticas de Promoción de } \\
\text { Salud y formulación de objetivos sani- } \\
\text { tarios para la década } 2000-2010 \\
\text { 2. Conocer la percepción de los } \\
\text { chilenos sobre su vida y salud, } \\
\text { incorporando aspectos de estado de } \\
\text { salud y factores condicionantes, para } \\
\text { los que no se cuenta con información } \\
\text { sistemática. } \\
\text { 3. Identificar inequidades en las condi- } \\
\text { ciones de salud } \\
\text { 4. Evaluar los resultados e impacto de } \\
\text { las políticas, planes y programas de } \\
\text { salud y realizar ajustes a las iniciativas } \\
\text { del sector en estas materias }\end{array}$ & $\begin{array}{l}\text { 1. Dolor permanente o frecuente den- } \\
\text { tro de los últimos } 12 \text { meses en "Cara, } \\
\text { boca y dientes" } \\
\text { 2. Dolor agudo (últimas } 2 \text { semanas) } \\
\text { asociado a "problema dental" } \\
\text { 3. Dificultad para el habla, mastica- } \\
\text { ción y alimentación }\end{array}$ \\
\hline $\begin{array}{l}\text { Segunda } \\
\text { Encuesta } \\
\text { Nacional de } \\
\text { Calidad de } \\
\text { Vida y Salud, } \\
2006\end{array}$ & $\begin{array}{l}\text { Encuesta } \\
\text { poblacional } \\
\text { de tipo trans- } \\
\text { versal. }\end{array}$ & $\begin{array}{l}\text { Personas }<15 \\
\text { años ( } n=4997, \\
3029 \text { hogares) } \\
\text { Personas } \geq 15 \\
\text { años ( } n=6130 \\
6168 \text { hogares) }\end{array}$ & $\begin{array}{l}\text { MINSAL INE } \\
\text { PUC }\end{array}$ & 2006 & $\begin{array}{l}\text { Informe } \\
\text { técnico }\end{array}$ & $\begin{array}{l}\text { Disponer de información sistemática, } \\
\text { confiable y oportuna acerca de la } \\
\text { calidad de vida y de la salud de la } \\
\text { población chilena; para el diseño, } \\
\text { desarrollo y evaluación de las políticas } \\
\text { e intervenciones de salud }\end{array}$ & $\begin{array}{l}\text { 1. Daño en salud y discapacidad } \\
\text { asociado a "problemas dentales" en } \\
\text { las últimas } 2 \text { semanas } \\
\text { 2. Impacto de la salud oral en la } \\
\text { calidad de vida } \\
\text { 3. Limitaciones en la vida diaria } \\
\text { relacionadas con el habla o con la } \\
\text { masticación de alimentos duros }\end{array}$ \\
\hline 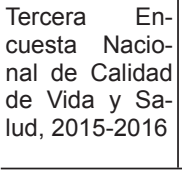 & $\begin{array}{l}\text { Encuesta po- } \\
\text { blacional de } \\
\text { tipo transver- } \\
\text { sal. }\end{array}$ & $\begin{array}{l}\text { Personas } \geq 15 \\
\text { años }(n=7041)\end{array}$ & $\begin{array}{l}M \text { I N S A } \\
P \cup C\end{array}$ & 2015- 2016 & $\begin{array}{l}\text { Informe téc- } \\
\text { nico }\end{array}$ & $\begin{array}{l}\text { Describir la percepción de los chilenos } \\
\text { mayores de } 15 \text { años en cuanto a su sa- } \\
\text { lud, calidad de vida y entorno para el } \\
\text { diseño, desarrollo y evaluación de las } \\
\text { políticas públicas e intervenciones en } \\
\text { salud. }\end{array}$ & $\begin{array}{l}\text { 1. Impacto de la salud oral en la cali- } \\
\text { dad de vida } \\
\text { 2. Impacto de la salud oral en la cali- } \\
\text { dad de vida a través de OHIP-7 (alto/ } \\
\text { bajo) }\end{array}$ \\
\hline
\end{tabular}

Tabla 2. Encuesta Nacional de Salud 2003, ENS 2009 - 2010, ENS 2016 - 2017.

\begin{tabular}{|c|c|c|c|c|c|c|c|}
\hline Nombre & $\begin{array}{l}\text { Tipo de } \\
\text { estudio }\end{array}$ & $\begin{array}{c}\text { Población } \\
\text { objetivo }\end{array}$ & Autor & $\begin{array}{c}\text { Año de } \\
\text { desarrollo }\end{array}$ & $\begin{array}{c}\text { Tipo de } \\
\text { publicación }\end{array}$ & Objetivo & Evento de salud oral \\
\hline $\begin{array}{l}\text { Encuesta } \\
\text { Nacional de } \\
\text { Salud, } 2003\end{array}$ & $\begin{array}{l}\text { Encuesta } \\
\text { poblacional } \\
\text { de tipo } \\
\text { transversal. }\end{array}$ & $\begin{array}{l}\text { Personas } \\
\geq 17 \text { años } \\
(n=3619)\end{array}$ & $\begin{array}{l}\text { MINSAL } \\
\text { PUC }\end{array}$ & 2000 & $\begin{array}{l}\text { Informe } \\
\text { técnico }\end{array}$ & $\begin{array}{l}\text { Determinar la prevalencia de las } \\
\text { enfermedades prioritarias y su va- } \\
\text { riabilidad, en población adulta chilena }\end{array}$ & $\begin{array}{l}\text { 1. Prevalencia de caries cavitadas y } \\
\text { promedio de dientes cariados } \\
\text { 2. Desdentamiento } \\
\text { 3. Uso de prótesis } \\
\text { 4. Frecuencia de cepillado } \\
\text { 5. Prevalencia de uso de servicios } \\
\text { de atención dental y motivo de } \\
\text { consulta } \\
\text { 6. Incomodidad generada por dien- } \\
\text { tes o prótesis en interacción social } \\
\text { 7. Autoreporte de desdentamiento, } \\
\text { caries y enfermedad periodontal }\end{array}$ \\
\hline $\begin{array}{l}\text { Encuesta } \\
\text { Nacional de } \\
\text { Salud, 2009- } \\
2010\end{array}$ & $\begin{array}{l}\text { Encuesta } \\
\text { poblacional } \\
\text { de tipo } \\
\text { transversal. }\end{array}$ & $\begin{array}{l}\text { Personas } \\
\geq 15 \text { años }(n= \\
5412)\end{array}$ & $\begin{array}{l}\text { MINSAL } \\
\text { PUC } \\
\text { OSUAH }\end{array}$ & $2009-2010$ & $\begin{array}{l}\text { Informe } \\
\text { técnico }\end{array}$ & $\begin{array}{l}\text { Determinar la prevalencia de los } \\
\text { problemas de salud prioritarios en la } \\
\text { población adulta de Chile utilizando } \\
\text { cuestionarios, exámenes de laborato- } \\
\text { rio y mediciones biofisiológicas. }\end{array}$ & $\begin{array}{l}\text { 1. Prevalencia de uso de prótesis } \\
\text { dental } \\
\text { 2. Prevalencia de satisfacción con } \\
\text { la prótesis } \\
\text { 3. Autoreporte de necesidad percibi- } \\
\text { da de prótesis dental } \\
\text { 4. Prevalencia de uso de servicios } \\
\text { de atención dental }\end{array}$ \\
\hline $\begin{array}{l}\text { Encuesta } \\
\text { Nacional de } \\
\text { Salud, 2016- } \\
2017\end{array}$ & $\begin{array}{l}\text { Encuesta } \\
\text { poblacional } \\
\text { de tipo } \\
\text { transversal. }\end{array}$ & $\begin{array}{l}\text { Personas } \\
\geq 15 \text { años } \\
(n=6233)\end{array}$ & $\begin{array}{l}\text { MINSAL } \\
\text { PUC } \\
\text { OSUAH }\end{array}$ & $\begin{array}{l}2016- \\
2017\end{array}$ & $\begin{array}{l}\text { Informe } \\
\text { técnico }\end{array}$ & $\begin{array}{l}\text { Estimar la prevalencia de problemas } \\
\text { de salud prioritarios y sus determi- } \\
\text { nantes en una muestra nacional de } \\
\text { hogares, representativa de la pobla- } \\
\text { ción general chilena no institucionali- } \\
\text { zada de } 15 \text { y más años. }\end{array}$ & $\begin{array}{l}\text { 1. Prevalencia de caries cavitadas y } \\
\text { promedio de dientes cariados. } \\
\text { 2. Desdentamiento } \\
\text { 3. Uso de prótesis } \\
\text { 3. Prevalencia de uso de servicios } \\
\text { de atención dental y motivo } \\
\text { 4. Percepción de salud bucal } \\
\text { 5. Incomodidad generada por dien- } \\
\text { tes o prótesis en interacción social }\end{array}$ \\
\hline
\end{tabular}

generar insumos para la formulación de los objetivos sanitarios para la década 2000-2010, en apoyo al proceso de reforma sectorial. Además, apuntaba a a obtener información útil para identificar la influencia de aspectos socioeconómicos y culturales en la salud, y su asociación con la calidad de vida de la población.

La muestra estuvo constituida por 6228 personas $\geq 15$ años de la misma cantidad de hogares, con representatividad nacional.

Contempló un cuestionario base de calidad de vida y un módulo específico de salud, el cual se subdividía en dos partes: el Módulo Individual y el Módulo Familiar.

En relación a la salud oral, en el Módulo Individual, se incluyó la "Cara, boca y dientes" como una de las posibles ubicaciones de dolores permanentes o frecuentes dentro de los últimos 12 meses a la fecha de aplicación de la encuesta. Además, se buscaba analizar el grado de interferencia en las actividades cotidianas generado por ese malestar.

En el Módulo Familiar, se consultó sobre la presencia de dolor agudo 
en el último tiempo (2 semanas), definiendo como una de las causas el "Problema Dental". Además se indagó por la dificultad para el habla, masticación y alimentación en alguna persona del hogar.

b. Segunda Encuesta Nacional de Calidad de Vida y Salud 2006(6) (Tabla 1):

Debido a la necesidad continua de proveer información adecuada para la toma de decisiones, se desarrolló la segunda versión, cuyo enfoque, además de evaluar los diversos aspectos de la salud, también buscaba analizar distintos aspectos de la vida: entorno, factores de riesgo, condiciones laborales, entre otros. Una diferencia respecto a la versión anterior es el estudio acerca del cuidado de los niños y su primera infancia. De esta forma, el objetivo general de esta encuesta fue disponer de información sistemática, confiable y oportuna acerca de la calidad de vida y de la salud de la población chilena para el diseño, desarrollo y evaluación de las políticas e intervenciones de salud.

Este estudio fue realizado por el MINSAL en colaboración con el INE y la Pontificia Universidad Católica de Chile (PUC) durante el año 2006, abarcando de forma representativa la población nacional, tanto urbana como rural. La muestra estuvo constituida por 6130 personas $\geq 15$ años, y se obtuvo información sobre 4997 personas $<15$ años.

El formato de esta versión estuvo compuesto por tres módulos: Módulo de Caracterización de la Vivienda y el Hogar, Módulo Individual y Módulo Infantil.

Dentro del área referente al "Daño en salud y discapacidad", se consultó sobre los problemas de salud en las últimas dos semanas.

En el área específica de salud oral, ésta se abordó a través de la frecuencia con que genera un impacto negativo en la calidad de las personas.

En el tópico de "Limitación de las Actividades de la Vida Diaria", se establecieron como opciones las limitaciones para hablar y para masticar alimentos duros (como manzanas o zanahorias).

c. Tercera Encuesta Nacional de Calidad de Vida y Salud 2015-2016 (79) (Tabla 1):

Para la tercera versión de esta encuesta, se mantuvieron los objetivos tradicionales, pero también se incorporaron nuevos instrumentos para la medición de la calidad de vida y sus diferentes aristas. Fue realizada en los años 2015-2016, siendo desarrollada por el MINSAL y la PUC. La muestra estuvo constituida por 7041 personas $\geq 15$ años.

Su objetivo general fue describir la percepción en cuanto a su salud, calidad de vida y entorno para el diseño, desarrollo y evaluación de las políticas públicas e intervenciones en salud.

Constaba de 10 módulos: Caracterización del encuestado, salud y trabajo, percepción de bienestar y salud, entorno y participación, enfermedades y accidentes, hábitos, apoyo social, sexualidad, educación y ocupación de los integrantes del hogar y características del hogar.

En el área específica de salud oral, se abordó el impacto del estado de los dientes y las encías en la calidad de vida.

Además, se realizó un índice Oral Health Impacto Profile (OHIP)-7, en donde se analizó el impacto (alto/ bajo) de la salud bucal en la calidad de vida. El instrumento OHIP-7 constaba de 7 ítems relacionados con la frecuencia de impacto adverso causado por la condición de salud oral durante los últimos 12 meses. Los ítems describían las siguientes dimensiones: limitación de funcionalidad, malestar psicológico, discapacidad física, discapacidad psicológica, discapacidad social, dolor físico y minusvalía (2).

d. Encuesta Nacional de Salud 2003(10-12) (Tabla 2):

La ENS 2003 fue desarrollada en conjunto por el MINSAL y la PUC, siendo un examen de salud voluntario, orientado a la población mayor de 17 años de todo el país. Se usó como base a las personas que participaron en la ENCAVI 2000.

La muestra estuvo constituida por 3619 personas, obteniéndose los datos directamente en su domicilio por una enfermera universitaria y un encuestador. Se indagó sobre signos o síntomas de algunas de las enfermedades más prevalentes en la población, se realizaron mediciones fisiológicas (condición nutricional, presión arterial, examen de visión, audición, y examen bucodental) y se obtuvieron muestras de sangre y orina. Se complementaron los datos con el autoreporte, cuestionarios, mediciones y exámenes de laboratorio para el estudio de las enfermedades más prevalentes.

Para la evaluación de la salud bucodental, se incluyó un módulo completo con preguntas relacionadas a la salud oral y a su impacto en la calidad de vida. Además, las enfermeras participantes recibieron capacitaciones sobre los siguientes tópicos: número de dientes remanentes en boca, presencia de caries cavitadas y uso de prótesis dental, a cargo de la unidad de Salud Dental del MINSAL y los equipos odontológicos regionales. Esta metodología fue evaluada con el piloto de la ENS, obteniendo sobre un $70 \%$ de concordancia con los diagnósticos realizados por cirujanos-dentistas.

Para la evaluación del desdentamiento, se consideraron $\geq 14$ dientes por arcada como cantidad total, constituyendo un mínimo de 28 dientes para dentados completos. Además, se definió como dentición no funcional, la presencia de $<20$ dientes en boca. También se consultó sobre el uso de prótesis dentales.

Para el análisis de prevalencia de dientes con caries cavitadas, se establecieron como parámetro mínimo 0 dientes cavitados y como máximo 21 dientes con caries cavitadas.

También se preguntó sobre la frecuencia de cepillado, así como el autoreporte de enfermedades orales.

e. Encuesta Nacional de Salud, 2009-2010(13-15) (Tabla 2):

Para que la información sobre enfermedades crónicas y sus determinantes esté disponible en forma oportuna y preste utilidad técnica, se requiere mantener una periodicidad en este tipo de estudios y garantizar su financiamiento. Por esto, el MINSAL decidió realizar la segunda versión de la Encuesta Nacional de Salud, en colaboración con PUC y el Observatorio Social de la Universidad Alberto Hurtado (OSUAH). Su objetivo general fue determinar la prevalencia de los problemas de salud prioritarios en la población adulta de Chile utilizando cuestionarios, exámenes de laboratorio y mediciones biofisiológicas.

La muestra estuvo constituida por 5434 personas $\geq 15$ años. Se realizaron exámenes y mediciones clínicas a 5043 participantes, y 4956 recibieron exámenes de laboratorio, teniendo representatividad nacional y regional.

La salud bucal fue evaluada a través de un cuestionario, sin realizar un examen clínico.

f. Encuesta Nacional de Salud, 2016-2017(12,16-20) (Tabla 2):

La Encuesta Nacional de Salud realizada entre los años 2016 y 2017, corresponde a la tercera y última versión de la encuesta poblacional de mayor envergadura en nuestro país, orientada al levantamiento de información para el desarrollo e implementación de políticas públicas para mejorar la salud de los chilenos en los años venideros. Su objetivo general fue estimar la prevalencia de problemas de salud prioritarios y sus determinantes en una muestra nacional de hogares representativa de la población general chilena no institucionalizada de 15 y más años.

La muestra estuvo constituida por 6233 personas $\geq 15$ años, de los cuales, 5520 contaban con examenes de laboratorio según protocolo. Tuvo representatividad nacional y regional. En la primera visita, se realizó la encuesta, y en una segunda visita, enfermeras realizaron mediciones antropométricas y toma de exámenes a la persona seleccionada, incluido un examen bucal, para lo que fueron previamente capacitadas, tal como sucedió en la versión inicial de la ENS 2003.

\section{Medición objetiva de las patologías orales más prevalentes} (Tabla 3)

\section{a. Caries dental:}

El año 2003, se reportó que promedio nacional de dientes con lesiones de caries cavitadas era de 2.52. Los hombres tenían significativamente más dientes cavitados por caries que las mujeres. El peak del promedio fue a los 25-44 años. Además, se observó una tendencia lineal a la baja en la medida que aumentaba el nivel socioeconómico y educacional. En zonas rurales, el promedio de dientes cavitados por caries fue casi dos veces mayor que en zonas urbanas. Al establecer la relación entre dientes con caries cavitadas y dientes remanentes, se observó un promedio de 0.15 , el cual va aumentando de manera lineal con la edad. Al mismo tiempo, la prevalencia de al menos un diente con caries cavitada fue de un $70.0 \%$, siendo levemente mayor en hombres que en mujeres, y aumentando con la edad hasta alcanzar un máximo entre los $25-44$ años, para luego disminuir gradualmente. Los niveles socioeconómicos y educacionales más bajos, así como en zonas rurales, presentan mayores prevalencias de caries dental(10-12).

En los años 2016-2017, la prevalencia de al menos un diente con caries cavitada fue de un $54.6 \%$, siendo levemente mayor en hombres que en mujeres, y aumentando con la edad hasta alcanzar un máximo entre los 45-64 años, para luego disminuir gradualmente. Los sujetos con $<8$ años de estudios, presentaban una mayor prevalencia de caries dental. Al comparar la prevalencia nacional con la ENS 2003, se observó una reducción en las caries cavitadas de un $15.4 \%$.

\section{b. Desdentamiento:}

El año 2003 , el $27.8 \%$ de la población de nuestro país eran dentados completos, siendo la frecuencia levemente mayor en hombres que en mujeres. Es importante destacar, que sin distinción de maxilar o sexo, sobre los 45 años el porcentaje de personas con dentición completa se redujo drásticamente bajo el $10 \%$. Otro fenómeno encontrado, es que al analizar el nivel socioeconómico, se observa que a medida que éste disminuye el porcentaje de personas con dentición completa disminuye notoriamente ${ }^{(10-12)}$. En los años 2016 - 2017, los sujetos dentados 
Tabla 3. Prevalencia de caries dental, desdentamiento y uso de prótesis parcial removible en ENCAVI 2000, ENCAVI 2006, ENCAVI 2015 - 2016, ENS 2003, ENS 2009 - 2010, ENS 2016 - 2017.

\begin{tabular}{|c|c|c|c|c|c|c|c|}
\hline Evento & Categoría & ENCAVI, 2000 & ENCAVI, 2006 & $\begin{array}{c}\text { ENCAVI, 2015- } \\
2016\end{array}$ & ENS, 2003 & ENS, 2009-2010 & ENS, 2016-2017 \\
\hline \multirow[b]{4}{*}{$\begin{array}{l}\text { Prevalencia caries } \\
\text { cavitadas }\end{array}$} & Total & NR & NR & NR & $70.0 \%$ & NR & $54.6 \%$ \\
\hline & \multirow{2}{*}{ Género } & \multirow{2}{*}{ NR } & \multirow{2}{*}{ NR } & \multirow{2}{*}{ NR } & Hombre: $71.2 \%$ & \multirow{2}{*}{ NR } & Hombre: $58.4 \%$ \\
\hline & & & & & Mujer: $68.8 \%$ & & Mujer: $50.6 \%$ \\
\hline & Edad & NR & NR & NR & $\begin{array}{l}\text { 17-24 años: } 56.4 \% \\
\text { 25-44 años: } 75.5 \% \\
45-64 \text { años: } 67.1 \% \\
\geq 65 \text { años: } 44.7 \%\end{array}$ & NR & $\begin{array}{l}\text { 15-24 años: } 40.9 \% \\
\text { 25-44 años: } 57.1 \% \\
45-64 \text { años: } 59.6 \% \\
\geq 65 \text { años: } 57.2 \%\end{array}$ \\
\hline $\begin{array}{l}\text { Promedio de dientes } \\
\text { cariados }\end{array}$ & Total & NR & NR & NR & 2.5 & NR & NR \\
\hline $\begin{array}{l}\text { Número de dientes } \\
\text { presentes }\end{array}$ & Total & NR & NR & NR & 21.43 dientes & NR & NR \\
\hline \multirow[b]{3}{*}{ Dentición completa } & Total & NR & NR & NR & $27.8 \%$ & NR & NR \\
\hline & Género & NR & NR & NR & $\begin{array}{c}\text { Hombre: } 29.2 \% \\
\text { Mujer: } 27.8 \%\end{array}$ & NR & NR \\
\hline & Edad & NR & NR & NR & $\begin{array}{c}17-24 \text { años: } 61.8 \% \\
25-44 \text { años: } 32.0 \% \\
45-64 \text { años: } 7.4 \% \\
\geq 65 \text { años: } 0.7 \%\end{array}$ & NR & $\begin{array}{c}15-24 \text { años: } 68.1 \% \\
\text { 25-34 años: } 59.6 \% \\
\text { 35-44 años: } 30.6 \% \\
\text { 45-54 años: } 13.0 \% \\
\text { 55-64 años: } 7.8 \% \\
\text { 65-74 años: } 2.6 \% \\
\geq 75 \text { años: } 0.9 \% \\
\end{array}$ \\
\hline \multirow[b]{3}{*}{ Desdentamiento parcial } & Total & NR & NR & NR & NR & NR & $\begin{array}{c}\mathrm{NR} \\
\end{array}$ \\
\hline & Género & NR & NR & NR & NR & NR & \begin{tabular}{|l|} 
NR \\
\end{tabular} \\
\hline & Edad & NR & NR & NR & NR & NR & $\begin{array}{l}\text { 15-24 años: } 31.9 \% \\
\text { 25-34 años: } 40.4 \% \\
\text { 35-44 años: } 69.0 \% \\
45-54 \text { años: } 85.6 \% \\
55-64 \text { años: } 86.0 \% \\
65-74 \text { años: } 79.8 \% \\
\geq 75 \text { años: } 57.8 \% \\
\end{array}$ \\
\hline \multirow[b]{3}{*}{ Dentición no funcional } & Total & NR & NR & NR & $29.7 \%$ & NR & $24.8 \%$ \\
\hline & Género & NR & NR & NR & $\begin{array}{c}\text { Hombre: } 25.0 \% \\
\text { Mujer: } 34.2 \% \\
\end{array}$ & NR & $\begin{array}{c}\text { Hombre: } 20.9 \% \\
\text { Mujer: } 29.1 \% \\
\end{array}$ \\
\hline & Edad & NR & NR & NR & NR & NR & $\begin{array}{c}\text { 15-24 años: } 0.4 \% \\
\text { 25-44 años: } 4.7 \% \\
45-64 \text { años: } 41.5 \% \\
\geq 65 \text { años: } 77.5 \% \\
\end{array}$ \\
\hline \multirow[b]{3}{*}{ Desdentamiento total } & Total & NR & NR & NR & $5.5 \%$ & NR & NR \\
\hline & Género & NR & NR & NR & $\begin{array}{c}\text { Hombre: } 3.3 \% \\
\text { Mujer: } 7.7 \% \\
\end{array}$ & NR & NR \\
\hline & Edad & NR & NR & NR & NR & NR & $\begin{array}{c}\text { 15-24 años: } 0.0 \% \\
\text { 25-34 años: } 0.0 \% \\
\text { 35-44 años: } 0.4 \% \\
45-54 \text { años: } 1.4 \% \\
55-64 \text { años: } 6.2 \% \\
\text { 65-74 años: } 17.6 \% \\
\geq 75 \text { años: } 41.2 \% \\
\end{array}$ \\
\hline \multirow[b]{3}{*}{ Uso de prótesis } & Total & NR & NR & NR & $25.0 \%$ & $22.9 \%$ & NR \\
\hline & Género & NR & NR & NR & $\begin{array}{c}\text { Hombre: } 19.0 \% \\
\text { Mujer: } 30.0 \%\end{array}$ & NR & $\begin{array}{c}\text { Hombre: } 15.6 \% \\
\text { Mujer: } 29.1 \%\end{array}$ \\
\hline & Edad & NR & NR & NR & NR & NR & $\begin{array}{c}\text { 15-24 años: } 0.2 \% \\
\text { 25-44 años: } 6.4 \% \\
45-64 \text { años: } 36.8 \% \\
\geq 65 \text { años: } 65.8 \% \\
\end{array}$ \\
\hline
\end{tabular}

ENCAVI: Encuesta Nacional de Calidad de Vida y Salud

ENS: Encuesta Nacional de Salud

Dentición no funcional: $<20$ dientes

Dentición completa: $\geq 14$ dientes por maxilar

NR: No reporta

completos disminuían gradualmente a medida que aumentaba la $\operatorname{edad}^{(16,17,19,20)}$.

En el año 2003, al evaluar la situación de las personas desdentadas parciales, la prevalencia de dentición no funcional, es decir, la presencia de $<20$ dientes en boca, fue de un $29.7 \%{ }^{(16)}$. Al analizar la cantidad de dientes por maxilar, la prevalencia de $\leq 10$ dientes en el maxilar fue de un $36.9 \%$, y en mandíbula, de un $30.7 \%$. El número promedio de dientes remanentes fue de 21.4, siendo mayor en los grupos de edad más jóvenes, hombres, nivel socioeconómico y educacional alto y zonas urbanas ${ }^{(10-12)}$. En los años 2016 - 2017, la prevalencia de dentición no funcional fue de un $24.8 \%$, siendo mayor en mujeres y al aumentar la edad. Al comparar el resultado nacional y en mujeres, éste fue significativamente menor que en la ENS 2003 ${ }^{(12,16-20)}$.

$\mathrm{Al}$ analizar la prevalencia de personas desdentadas totales, el año 2003 , el $5.5 \%$ de la población era desdentada bimaxilar, porcentaje que aumentaba drásticamente a un $33.4 \%$ en las personas $\geq 65$ años. El nivel socioeconómico y educacional también marcaban tendencia en el grado de desdentamiento, presentando mayores prevalencias en los niveles de ingresos y educación más bajos ${ }^{(10-12)}$. En el año 2016 - 2017, la prevalencia de desdentados totales alcanzaba a un $17.6 \%$ en los sujetos 
$\geq 65$ años y un $41.2 \%$ en el grupo $\geq 75$ años $(16,17,19,20)$.

c. Uso de prótesis dental:

El año 2003 , el $25 \%$ de la población utilizaba prótesis dental, con diferencia entre el uso de prótesis maxilares $(15.4 \%)$ en comparación al uso de prótesis mandibulares $(0.7 \%)$. El porcentaje de personas que usaba prótesis dental aumentaba progresivamente con la edad, y en las clases socioeconómicas y educacional bajas ${ }^{(10-12)}$. En los años $2009-$ 2010, la prevalencia de uso de prótesis dental (autoreportada) fue de un $22.9 \%^{(13-15)}$. En los años $2016-2017$, el $22.4 \%$ de la población utilizaba prótesis dental, donde el uso de prótesis maxilar era de un $13.4 \%$, y del $1.2 \%$ para la mandibular. Se observaron diferencias significativas tanto para el sexo, la edad y los años totales de estudios cursados, donde la mayor prevalencia de uso de prótesis fue en las mujeres, en los $\geq 65$ años y los que habían cursado $<8$ años de educación ${ }^{(12,16-20)}$.
3. Medición subjetiva del impacto de las patologías orales en la calidad de vida relacionada con la salud oral (Tabla 4):

a. Prevalencia de problemas dentales durante las 2 semanas previas a la encuesta:

En el año 2000, se reportó que la "Cara, boca o dientes" no representaban una zona que generara dolores permanentes o frecuentes. Sin embargo, los problemas dentales como causa de episodios de dolor agudo durante las últimas 2 semanas, presentaban una prevalencia de un $3.52 \%$, siendo mayor en las mujeres $(4.1 \%$ versus $2.9 \%$ en hombres) y en los sujetos de 15-19 años (4.6\%). La incidencia según género, cada 100 habitantes, fue de 4 en mujeres y de 3 en hombres ${ }^{(4)}$. Si solo se analizan los problemas de salud de las últimas 2 semanas, los problemas dentales, en el año 2006, ocupaban el sexto lugar de mayor prevalencia, con un $22.6 \%$. Esto posiciona a los problemas dentales como una

Tabla 4. Medición subjetiva del impacto de las patologías orales en la calidad de vida relacionada con la salud en ENCAVI 2000, ENCAVI 2006, ENCAVI 2015 - 2016, ENS 2003, ENS 2009 - 2010, ENS 2016 - 2017.

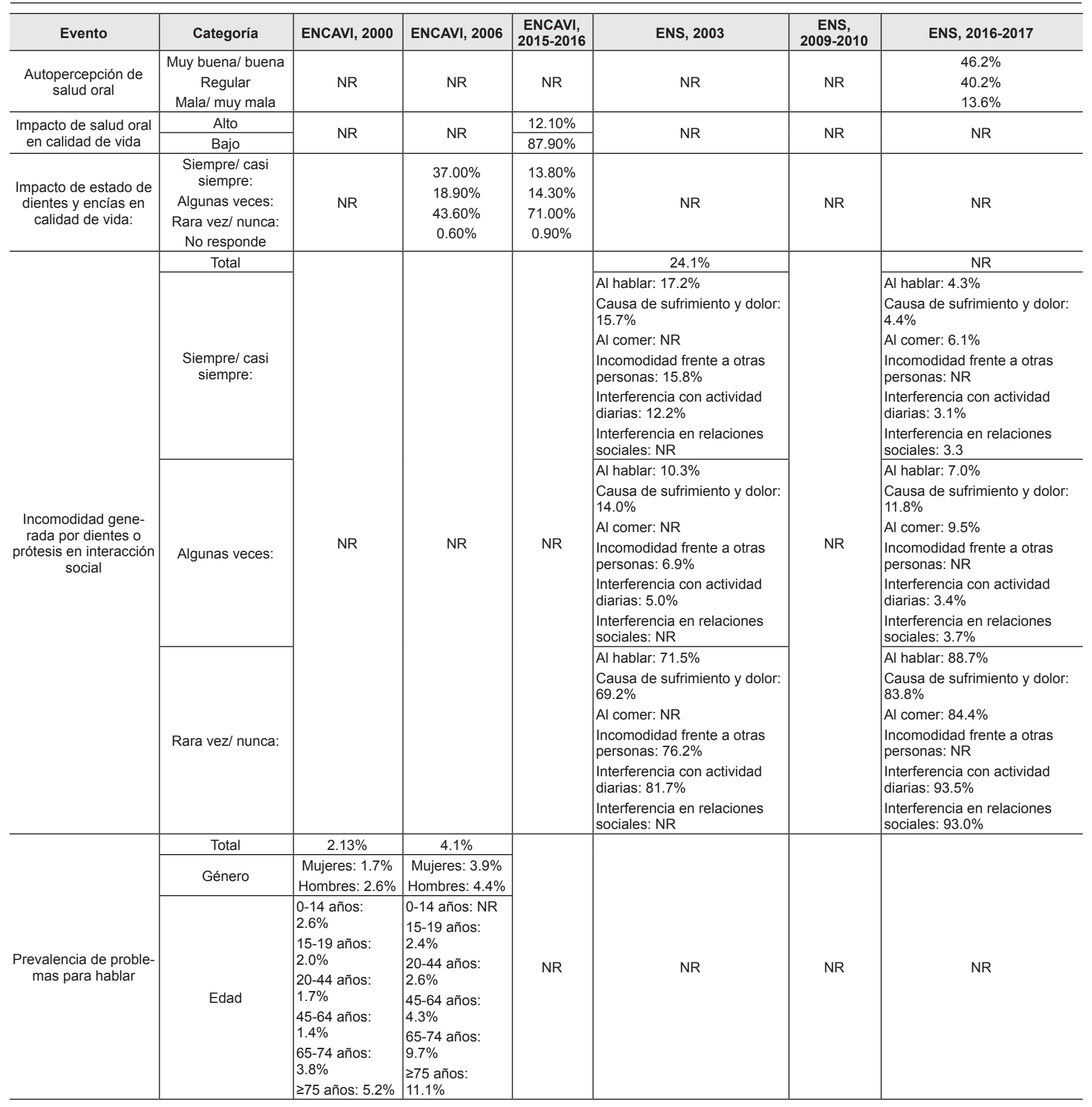


Tabla 4. Medición subjetiva del impacto de las patologías orales en la calidad de vida relacionada con la salud en ENCAVI 2000, ENCAVI 2006, ENCAVI 2015 - 2016, ENS 2003, ENS 2009 - 2010, ENS 2016 - 2017. (continuación)

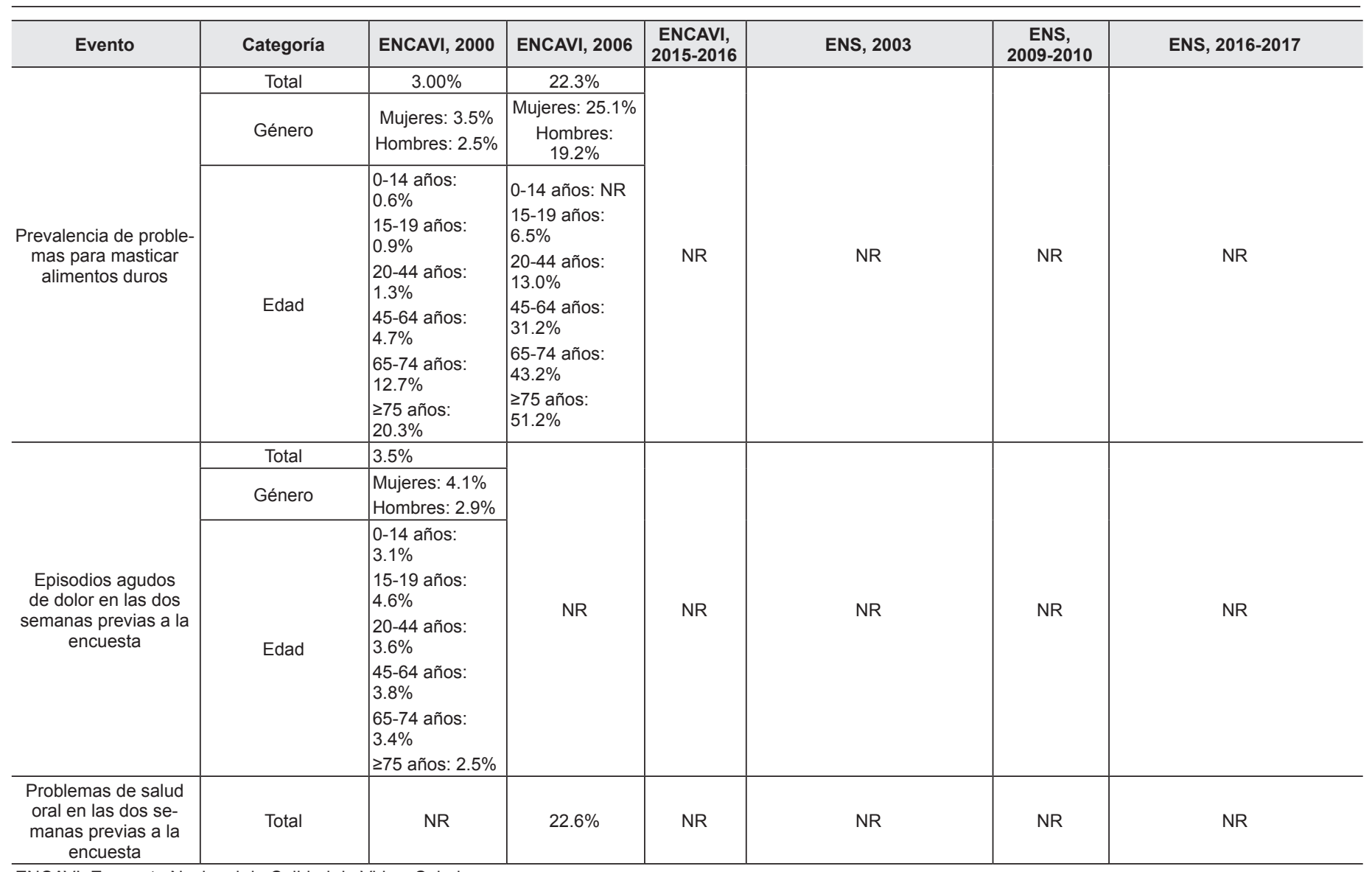

ENCAVI: Encuesta Nacional de Calidad de Vida y Salud

ENS: Encuesta Nacional de Salud

NR: No reporta

Tabla 5. Uso de servicio dental en ENCAVI 2000, ENCAVI 2006, ENCAVI 2015-2016, ENS 2003, ENS 2009-2010, ENS 2016-2017

\begin{tabular}{|c|c|c|c|c|c|c|c|}
\hline Evento & Categoría & $\begin{array}{c}\text { ENCAVI, } \\
2000\end{array}$ & $\begin{array}{l}\text { ENCAVI, } \\
2006\end{array}$ & $\begin{array}{l}\text { ENCAVI, } \\
\text { 2015-2016 }\end{array}$ & ENS, 2003 & ENS, 2009-2010 & ENS, 2016-2017 \\
\hline \multirow{6}{*}{$\begin{array}{l}\text { Prevalencia de } \\
\text { uso de servicio } \\
\text { dental }\end{array}$} & $<6$ meses & \multirow{6}{*}{ NR } & \multirow{6}{*}{ NR } & \multirow{6}{*}{ NR } & $23.8 \%$ & $23.6 \%$ & \multirow{2}{*}{$45.0 \%$} \\
\hline & 6 meses- 1 año & & & & $14.7 \%$ & $20.4 \%$ & \\
\hline & 1- 2 años & & & & $18.6 \%$ & $282 \%$ & \multirow{3}{*}{$52.8 \%$} \\
\hline & 2- 5 años & & & & $15.8 \%$ & 20.210 & \\
\hline & $\geq 5$ años & & & & $23.2 \%$ & $23.5 \%$ & \\
\hline & Nunca & & & & $3.9 \%$ & $4.3 \%$ & $2.2 \%$ \\
\hline \multirow{6}{*}{$\begin{array}{l}\text { Motivo de consul- } \\
\text { ta al odontológo }\end{array}$} & Dolor o sospecha de caries & \multirow{6}{*}{ NR } & \multirow{6}{*}{ NR } & \multirow{6}{*}{ NR } & $35.5 \%$ & \multirow{6}{*}{ NR } & $28.6 \%$ \\
\hline & Sangrado de encías & & & & $2.3 \%$ & & NR \\
\hline & Control/ continuar tratamiento & & & & $21.5 \%$ & & $66.9 \%$ \\
\hline & Estética (prótesis u ortodoncia) & & & & $17.2 \%$ & & NR \\
\hline & Otra razón & & & & $20.1 \%$ & & NR \\
\hline & Nunca lo ha visitiado/ No responde & & & & $3.4 \%$ & & $4.5 \%$ \\
\hline
\end{tabular}

ENCAVI: Encuesta Nacional de Calidad de Vida y Salud

ENS: Encuesta Nacional de Salud

NR: No reporta

alteración común y presenta mayor prevalencia que episodios de fiebre, depresión, pérdida de equilibrio y enfermedades cutáneas ${ }^{(6)}$.

b. Problemas para hablar y masticar:

En el año $2000^{(4)}$, el $2.1 \%$ de la población señaló tener dificultades para hablar, aumentando a un $4.1 \%$ en el año $2006^{(6)}$. Así mismo, la prevalencia de sujetos que relataban dificultades para masticar alimentos duros aumentó de un $3 \%$ en el año $2000^{(4)}$, a un $22.3 \%$ en el año $2006^{(6)}$.
Este incremento se observó en todos los grupos de edad.

c. Impacto de problemas orales en calidad de vida relacionada con la salud:

En relación con la percepción de salud bucal de la población, los años 2016 - 2017, se describe que existe poca diferencia entre los porcentajes mayoritarios correspondientes a quienes consideraban su salud bucal "muy buena o buena" (46.2\%) y quienes la consideraban "regular" 
(40.2\%), donde solo un $13.2 \%$ consideraba que su salud oral es "mala o muy mala". La percepción "mala o muy mala" aumentó significativamente a medida que los sujetos tenían mayor edad, y menor cantidad de años de estudio(16,17,19,20).

En el año 2006, el $37.0 \%$ de los sujetos respondieron que el estado de sus dientes y encías afectaba casi siempre/ siempre su calidad de vida, mantendiéndose relativamente constante este valor a lo largo del ciclo

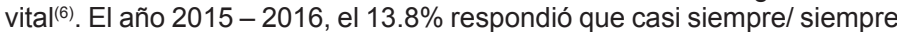
se veía impactada su calidad de vida por su salud oral. Esto aumentó a un $23.7 \%$ en los sujetos $\geq 65$ años. Además, se reportó que el $12.1 \%$ de los sujetos encuestados respondieron que su salud oral tenía un alto impacto en su calidad de vida(7-9).

El año 2003, se reportó que la prevalencia de incomodidad de dientes o prótesis fue de un $24.1 \%$, aumentando con la edad, y siendo mayor en las mujeres y niveles socioeconómicos bajos ${ }^{(10-12)}$. Este resultado fue menor en los años $2016-2017^{(16,17,19,20)}$.

Finalmente, en los años 2009 - 2010, el $21.1 \%$ de los encuestados manifestó que se encontraba "poco conforme" o "nada conforme, no me sirve" con la prótesis dental. La necesidad autopercibida de uso de prótesis dental fue de un $25.3 \%$, siendo mayor en los sujetos de $45-64$ años $(44.3 \%)$ y $\geq 65$ años $(55.3 \%)$. Se observó una disminución significativa de la percepción de esta necesidad a medida que el nivel educacional aumentaba y en sujetos de zonas urbanas ${ }^{(13-15)}$.

\section{Uso de servicio dental (Tabla 5):}

El año 2003 , el $23.2 \%$ de los sujetos consultó al dentista hace más de 5 años, y el $3.9 \%$ nunca lo había visitado. Sin embargo, el $23.8 \%$ lo visitó hace menos de 6 meses. Las personas que no habían visto nunca al dentista aumentaban de un $3.3 \%$ en los jóvenes de 17-24 años a un $7.2 \%$ en los $\geq 65$ años. En relación al nivel socioeconómico y cultural, los estratos más altos presentaban un menor porcentaje de sujetos que nunca habían visitado al dentista versus los estratos más pobres. Lo mismo ocurre con las zonas urbanas versus las rurales(10-12). En los años 2009 - 2010, el $23.5 \%$ de los encuestados declararon no haber visitado al odontólogo durante los últimos 5 años, y el $4.3 \%$, no haberlo visitado nunca. Por otra parte, el porcentaje de personas que no visitaba al odontólogo en los últimos 5 años, fue mayor en los grupos de $45-64$ años, $\geq 65$ años, y en los estratos educacionales bajos ${ }^{(13-15)}$. Finalmente, en los años 2016 - 2017, un $45.0 \%$ refirió haber consultado al odontólogo hace $\leq 1$ año, $52.8 \%$ declaró $>1$ año, y un $2.2 \%$, declaró nunca haberlo visitado. Existían diferencias en relación al género, edad y años de educación, donde la mayor prevalencia de sujetos que habían asistido al dentista durante los últimos 12 meses fueron las mujeres, jóvenes de $15-24$ años y sujetos con $>12$ años de educación ${ }^{(16,17,19,20)}$.

Las razones más frecuentes para la visita al odontológo, en el año 2003 , fueron el dolor o la sospecha de caries (35.5\%), seguido por un control odontológico $(21.5 \%)$. En los adultos $\geq 65$ años, la causa más prevalente fue la prótesis dental $(50.9 \%)$. También hubo un gradiente socioeconómico en las visitas preventivas, donde en las clases altas, el $33.8 \%$ acudió a control o revisión, mientras en las clases más bajas, esto caía a un $8.7 \%$. Resultados similares se observaron al analizar el nivel educacional y las zonas urbanas versus las rurales ${ }^{(10-12)}$. En los años 2016 - 2017, la principal causa de visita al odontológo fue la continuación de tratamiento $(40.3 \%)$, seguido por el dolor o molestias en la boca, dientes o encías $(28.6 \%)$, y por control de rutina $(26.6 \%)^{(16,17,19,20)}$.

\section{DISCUSIÓN}

La salud oral es una parte integral del bienestar de las personas, familias y comunidades. La calidad de vida relacionada con la salud oral ha mejorado en los últimos años debido a la contribución de las políticas y programas de salud bucal mediante estrategias sectoriales, intersectoriales y participativas para la promoción de la salud, prevención de enfermedades bucales y tratamiento odontológico. Es así como se observa una disminución en las personas que declararon que el estado de sus dientes/ encías/ prótesis impactaban negativamente siempre o casi siempre su capacidad de hablar, comer, que le causaban sufrimiento o interferían en sus actividades diarias desde el año $2003^{(10)}$ al 2017(16).

La prevalencia de caries cavitadas disminuyó significativamente de un $70.0 \%{ }^{(10)}$ a un $54.6 \%^{(16)}$ desde el año 2003 al 2017. Lo mismo ocurrió con la prevalencia de dentición no funcional, la que se redujo de un $29.7 \%{ }^{(10)}$ a un $24.8 \%{ }^{(16)}$ en los mismos años. Sin embargo, si bien la primera causa de consulta odontológica pasó de ser el dolor/ sospecha de caries a continuación de tratamiento, los sujetos que no acudían al odontólogo en $>5$ años, se ha mantenido en las mismas proporciones en el mismo período de tiempo ${ }^{(10,15,16)}$. Además, se mantienen las inequidades en salud, donde se observa un gradiente socioeconómico en la prevalencia de caries, desdentamiento, acceso a la salud oral, prótesis y en el impacto de la salud oral en la calidad de vida.

Los cambios en las prevalencias de caries cavitadas y de dentición no funcional podrían explicarse por la incorporación de las Garantías Explícitas en Salud (GES) de "Salud oral integral para niñas y niños de 6 años" en el año 2005, del GES de "Atención odontológica integral adulto de 60 años" en el año 2007, y del GES de "Salud oral integral de la embarazada"(21) en el año 2010. Sumado a esto, el Programa Más Sonrisas para Chile ha mejorado el acceso a la atención odontológica de las mujeres mayores de 20 años, permitiéndoles recuperar su salud bucal cuando la han perdido y entregándoles las herramientas para cuidarla. El Programa de Mejoramiento del Acceso a la Atención Odontológica para los Jóvenes de Cuarto Año de Educación Media, de colegios municipales y particulares subvencionados, y Atención de Morbilidad Odontológica de los adultos, han acercado la atención a las personas que por sus actividades académicas o laborales tienen dificultad para acceder a los lugares y en los horarios habituales de atención clínica ${ }^{(22)}$.

Las encuestas descritas en esta revisión son las ENCAVI y ENS. Sin embargo, también se han realizado encuestas sobre el consumo de tabaco en jóvenes ${ }^{(23-26)}$ y en estudiantes de profesiones de la salud(27), así como encuestas de salud escolar(28,29). La Encuesta Mundial de Salud Escolar 2004-2005 fue aplicada solamente en las Regiones de Tarapacá, Valparaíso, Metropolitana y del Bío Bío en una muestra representativa de adolescentes de 13 a 15 años. El $85 \%$ de los encuestados de las Regiones Metropolitana y del Bío Bío declararon lavarse los dientes 2 o más veces al día. Entre el 1 y $2 \%$ refirió no haberse cepillado durante el último mes ${ }^{(28)}$. La segunda versión de esta encuesta no contempló la salud oral(29)

Lamentablemente, si bien los datos recopilados son diversos, no en todas las ocasiones se realizan las mismas mediciones ni se levanta la misma información, por lo que las comparaciones son difíciles de realizar. Además, no se ha incorporado el componente de salud oral en la evaluación de sujetos $<15$ años, por lo que no se tiene conocimiento sobre el impacto de la salud bucal en su calidad de vida ni tampoco la prevalencia de las enfermedades orales más prevalentes. Por otro lado, las ENS 2003 y 2016-2017 definen al personal de enfermería como evaluadores de la salud oral, lo que, en las etapas de capacitación, presentaban una concordancia con el diagnóstico de un odontólogo de un $70 \%$, lo que podría estar sobre o subestimando la prevalencia de las enfermedades orales. Sumado a esto, no se ha evaluado la prevalencia de las enfermedades periodontales, que sí se han reconocido como un problema de salud pública ${ }^{(30)}$ y enfermedades crónicas no transmisibles ${ }^{(31)}$. Al no tener información sobre los puntos anteriores, se dificulta la formulación de políticas públicas adecuadas a las necesidades nacionales.

\section{CONCLUSIONES}

Las encuestas nacionales de salud son consideradas herramientas indispensables para apoyar los procesos de decisión en salud pública y en la formulación de políticas públicas adecuadas a las necesidades nacionales. En Chile, la prevalencia de caries cavitada y dentición no funcional ha disminuido significativamente, y ha mejorado la calidad de vida relacionada con la salud oral. Sin embargo, se han mantenido las inequidades en salud. Además, las encuestas nacionales no son de enfermedades orales, ni se han realizado en niños y adolescentes, sugiriendo que se incorporen los puntos anteriores a las próximas encuestas poblacionales a realizarse en nuestro país o bien que se haga una encuesta nacional de salud oral.

\section{CONFLICTO DE INTERÉS}

Ninguno.

\section{FUENTE DE FINANCIAMIENTO}

Agencia Nacional de Investigación y Desarrollo / Programa Becas / Beca Doctorado Nacional/2018 - 21180023.

\section{RELEVANCIA CLÍNICA}

Existe una alta carga de enfermedades orales y dentición no funcional en la población adulta y adulta mayor chilena, lo que tiene un impacto en la calidad de vida de las personas. Sin embargo, las encuestas nacionales no son de enfermedades orales, ni se han realizado en niños y adolescentes, sugiriendo que se incorporen los puntos anteriores a las próximas encuestas poblacionales a realizarse en nuestro país o bien que se haga una encuesta nacional de salud oral. 


\section{Bibliografía}

1. Ministerio de Salud de Chile. Encuesta Nacional de Salud ENS Chile 2009-2010. I. Resumen ejecutivo y equipo de trabajo [Internet]. 2010 [consultado 10/02/2020]. Disponible en: http://epi.minsal.cl/resultados-encuestas/

2. Sabando V, Albala C. Calidad de vida relacionada con salud oral y autopercepción de salud: Encuesta Nacional de Calidad de Vida y Salud 2015-2016 en Chile. Int J Odontostomatol. 2019:13(3):338-44.

3. Ministerio de Salud de Chile. Encuesta Nacional de Salud 2016 - 2017. Primeros resultados [Internet]. 2017 [consultado 25/03/2020]. Disponible en: https://www. minsal.cl/wp-content/uploads/2017/11/ENS-2016-17_PRIMEROS-RESULTADOS. pdf

4. Ministerio de Salud de Chile, Instituto Nacional de Estadística. Primera Encuesta Nacional de Calidad de Vida y Salud [Internet]. 2016 [consultado 06/02/2020]. Disponible en: http://epi.minsal.cl/wp-content/uploads/2016/03/presentacion2.pdf

5. Gonzalez C, Aguilera X, Betancur C, Guerrero A, Salinas J, Donoso N. Encuesta de calidad de vida y salud, MINSAL-INE, 2000. EI Vigía. 2002;5(16):2-8.

6. Ministerio de Salud de Chile. II Encuesta de Calidad de Vida y Salud [Internet] 2016 [consultado 07/02/2020]. Disponible en: http://epi.minsal.cl/wp-content/ uploads/2016/03/ENCAVI.2006.pdf

7. Ministerio de Salud de Chile. Informe de Principales Resultados Tercera Versión de la Encuesta de Calidad de Vida y Salud ENCAVI 2015-2016 [Internet]. 2018 [consultado 07/02/2020]. Disponible en: http://epi.minsal.cl/wp-content/ uploads/2018/11/Encavi_2015_2016.pdf

8. Ministerio de Salud de Chile. Encuesta de Calidad de Vida y Salud (ENCAVI) 2015 - 2016. Resultados Abril 2017 [Internet]. 2017 [consultado 07/02/2020] Disponible en: http://epi.minsal.cl/wp-content/uploads/2017/06/Resultados_ Abril2017_ENCAVI_2015-16_Depto_Epidemiología_MINSAL.pdf

9. Ministerio de Salud de Chile. Resultados de la Tercera versión de la Encuesta de Calidad de Vida y Salud - ENCAVI 2015-2016 [Internet]. 2018 [consultado 07/02/2020]. Disponible en: http://epi.minsal.cl/encavi-2018/\#/

10. Ministerio de Salud de Chile. Resultados I Encuesta de Salud, Chile 2003 [Internet]. [consultado 07/02/2020]. Disponible en: http://epi.minsal.cl/wp-content/ uploads/2016/03/InformeFinalENS2003.vent_.pdf

11. Ministerio de Salud de Chile. Encuesta Nacional de Salud (2004) [Internet] [consultado 07/02/2020]. Disponible en: http://epi.minsal.cl/wp-content/ uploads/2016/03/presentacion.ens .vent .pdf

12. Ministerio de Salud de Chile. Fe de erratas [Internet]. 2019 [consultado 10/02/ 2020]. Disponible en: http://epi.minsal.cl/wp-content/uploads/2019/01/Fe-deerratas-2o-Resultados-ENS DEPTO.EPIDEMIOLOGIA.MINSAL.14.01.2019.pdf

13. Ministerio de Salud de Chile. Encuesta Nacional de Salud ENS Chile 2009 2010. II. Antecedentes [Internet]. 2010 [consultado 10/02/2020]. Disponible en: http://epi.minsal.cl/resultados-encuestas/

14. Ministerio de Salud de Chile. Encuesta Nacional de Salud ENS Chile 2009-2010. III. Metodología [Internet]. 2010 [consultado 10/02/2020]. Disponible en: http://epi. minsal.cl/resultados-encuestas/

15. Ministerio de Salud de Chile. Encuesta Nacional de Salud ENS Chile 2009 2010. V. Resultados [Internet]. 2010 [consultado 10/02/2020]. Disponible en: http:// epi.minsal.cl/resultados-encuestas/

16. Ministerio de Salud de Chile. Encuesta Nacional de Salud 2016-2017. Segunda entrega de resultados [Internet]. 2019 [consultado 10/02/2020]. Disponible en: http://epi.minsal.cl/wp-content/uploads/2019/01/2o-Resultados-ENS_DEPTO. EPIDEMIOLOGIA.MINSAL.14.01.2019.pdf

17. Ministerio de Salud de Chile. Documento segunda entrega de resultados. Tercera Encuesta Nacional de Salud (ENS) 2016-2017 [Internet]. 2019 [consultado 10/02/2020]. Disponible en: http://epi.minsal.cl/wp-content/ uploads/2019/01/MinutaTecnica.-2o-Resultados-ENS DEPTO.EPIDEMIOLOGIA MINSAL.14.01.2019.pdf
18. Ministerio de Salud de Chile. Encuesta Nacional de Salud 2016-2017. Objetivos. [Internet]. [consultado 10/02/2020]. Disponible en: http://epi.minsal.cl/wp-content/ uploads/2018/05/OBJETIVOS-ENS-2016-2017-.pdf

19. Ministerio de Salud de Chile. Sábana general de resultados (actualización 14.02.2019) [Internet]. 2019 [consultado 10/02/2020]. Disponible en: http://epi. minsal.cl/resultados-encuestas/

20. Mendoza C, Ministerio de Salud de Chile. Salud Bucal en las personas mayores: contribuyendo a un envejecimiento saludable [Internet]. 2019 [consultado 10/02/2020]. Disponible en: https://www.minsal.cl/wp-content/uploads/2019/06/ Dra.-Carolina-Mendoza-Salud-bucal-en-personas-mayores.pdf

21. Jara G, Werlinger F, Cabello R, Carvajal P, Espinoza I, Giacaman R, et al. Sinopsis de la situación de salud oral en Chile - Parte I: Garantías explícitas y guías clínicas. Rev clínica periodoncia, Implantol y Rehabil oral. 2018:11(3):187-90.

22. Ministerio de Salud de Chile. Plan Nacional de Salud Bucal 2018-2030 [Internet]. 2017 [consultado 11/02/2020]. Disponible en: https://www.minsal.cl/ wp-content/uploads/2017/12/Plan-Nacional-Salud-Bucal-2018-2030-ConsultaPública-20 12 2017.pdf

23. Ministerio de Salud de Chile, Universidad de Chile, OPS - OMS, CDC - Atlanta. Algunos resultados Encuesta Mundial de Tabaquismo en Jovenes. Chile 2000 (GYTS) [Internet]. 2016 [consultado 11/02/2020]. Disponible en: http://epi.minsal.cl/ wp-content/uploads/2016/03/EMTA.2000.pdf

24. Ministerio de Salud de Chile. Encuesta Mundial de Tabaquismo en Jovenes 2003 [Internet]. 2005 [consultado 11/02/2020]. Disponible en: http://epi.minsal.cl/wpcontent/uploads/2016/06/Encuesta mundial tabaquismo jovenes 2003.pdf

25. Ministerio de Salud de Chile. Encuesta Mündial de Tabaquismo én Adolescentes EMTA, Chile 2008 [Internet]. 2008 [consultado 11/02/2020]. Disponible en: http://epi. minsal.cl/wp-content/uploads/2016/03/EMTA20081.pdf

26. Ministerio de Salud de Chile, OPS - OMS. Informe Encuesta Mundial sobre Tabaco en Jovenes 2016 [Internet]. [consultado 11/02/2020]. Disponible en: http:// epi.minsal.cl/wp-content/uploads/2018/06/Informe-GYTS-final_junio2018.pdf

27. Ministerio de Salud de Chile. Encuesta Mundial de Tabaquismo en Estudiantes Profesiones de Salud (EMTES) [Internet]. 2008 [consultado 11/02/2020]. Disponible en: http://epi.minsal.cl/wp-content/uploads/2016/03/InformeFinalEMTESChile2008. pdf

28. Ministerio de Salud de Chile, OPS - OMS, CDC - Atlanta. Encuesta Mundial de Salud Escolar. Chile. 2004 y 2005 [Internet]. 2005 [consultado 11/02/2020]. Disponible en: http://epi.minsal.cl/wp-content/uploads/2016/03/EMSE.2004-2005final.pdf

29. Ministerio de Salud de Chile, OPS - OMS, CDC - Atlanta. Informe de la Encuesta Mundial de Salud Escolar. Chile 2013 [Internet]. 2016 [consultado 11/02/2020]. Disponible en: http://epi.minsal.cl/wp-content/uploads/2016/03/EMSE.2013.pdf

30. Carvajal P. Enfermedades periodontales como un problema de salud pública: el desafío del nivel primario de atención en salud. Rev Clínica Periodoncia, Implantol y Rehabil Oral [Internet]. 2016;9(2):177-83. Available from: http://dx.doi.org/10.1016/j. piro.2016.07.001

31. Morales A, Bravo J, Baeza M, Werlinger F, Gamonal J. Las enfermedades periodontales como enfermedades crónicas no transmisibles: cambios en los paradigmas. Rev Clin Periodoncia Implant Rehabil Oral. 2016;9(2):203-7. 